\title{
Discovery of X-ray emission from the young radio pulsar PSR J1357-6429
}

\author{
P. Esposito ${ }^{1,2}$, A. Tiengo ${ }^{2}$, A. De Luca ${ }^{2}$, and F. Mattana ${ }^{2,3}$ \\ 1 Università degli Studi di Pavia, Dipartimento di Fisica Nucleare e Teorica and INFN-Pavia, via Bassi 6, 27100 Pavia, Italy \\ e-mail: paoloesp@iasf-milano.inaf.it \\ 2 INAF - Istituto di Astrofisica Spaziale e Fisica Cosmica Milano, via Bassini 15, 20133 Milano, Italy \\ 3 Università degli Studi di Milano - Bicocca, Dipartimento di Fisica G. Occhialini, p.za della Scienza 3, 20126 Milano, Italy
}

Received 14 March 2007 / Accepted 10 April 2007

ABSTRACT

\begin{abstract}
We present the first X-ray detection of the very young pulsar PSR J1357-6429 (characteristic age of 7.3 kyr) using data from the XMM-Newton and Chandra satellites. We find that the spectrum is well described by a power-law plus blackbody model, with photon index $\Gamma=1.4$ and blackbody temperature $k_{\mathrm{B}} T=160 \mathrm{eV}$. For the estimated distance of $2.5 \mathrm{kpc}$, this corresponds to a $2-10 \mathrm{keV}$ luminosity of $\sim 1.2 \times 10^{32} \mathrm{erg} \mathrm{s}^{-1}$, thus the fraction of the spin-down energy channeled by PSR J1357-6429 into X-ray emission is one of the lowest observed. The Chandra data confirm the positional coincidence with the radio pulsar and allow to set an upper limit of $3 \times 10^{31} \mathrm{erg} \mathrm{s}^{-1}$ on the $2-10 \mathrm{keV}$ luminosity of a compact pulsar wind nebula. We do not detect any pulsed emission from the source and determine an upper limit of $30 \%$ for the modulation amplitude of the X-ray emission at the radio frequency of the pulsar.
\end{abstract}

Key words. stars: individual: PSR J1357-6429 - stars: neutron - X-rays: stars

\section{Introduction}

X-ray observations of radio pulsars provide a powerful diagnostic of the energetics and emission mechanisms of rotationpowered neutron stars. Due to the magnetic dipole braking, a pulsar loses rotational kinetic energy at a rate $\dot{E}=4 \pi^{2} I \dot{P} P^{-3}$, where $I$ is the moment of inertia of the neutron star, assumed to be $10^{45} \mathrm{~g} \mathrm{~cm}^{2}$, and $P$ is the rotation period. Though pulsars have traditionally been mostly studied at radio wavelengths, only a small fraction $\left(10^{-7}\right.$ to $10^{-5}$, e.g., Taylor et al. 1993) of the "spin-down luminosity" $\dot{E}$ emerges as radio pulsations. Rotation power can manifest itself in the $\mathrm{X} / \gamma$-ray energy range as pulsed emission, or as nebular radiation produced by a relativistic wind of particles emitted by the neutron star. Residual heat of formation is also observed as soft X-ray emission from young neutron stars. Such thermal radiation, however, can also be produced as a result of reheating from internal or external sources. The growing list of observable X-ray emitting rotation-powered pulsars allows the study of the properties of the population as a whole. Young pulsars constitute a particularly interesting subset to investigate owing to their large spin-down luminosities $\left(\gtrsim 10^{36} \mathrm{erg} \mathrm{s}^{-1}\right)$.

The discovery of PSR J1357-6429 during the Parkes multibeam survey of the Galactic plane (see Lorimer et al. 2006, and references therein) is reported in Camilo et al. (2004). The pulsar is located near the supernova remnant candidate G309.8-2.6 (Duncan et al. 1997) for which no distance or age information is available. With a spin period of $166 \mathrm{~ms}$, a period derivative of $3.6 \times 10^{-13} \mathrm{~s} \mathrm{~s}^{-1}$, and a characteristic age $\tau_{\mathrm{c}}=P / 2 \dot{P} \simeq 7300 \mathrm{yr}$, this pulsar stands out as one of the ten youngest Galactic radio pulsars known (see the ATNF Pulsar Catalogue ${ }^{1}$, Manchester et al. 2005). The other main properties of this source derived

\footnotetext{
${ }^{1}$ See http://www.atnf.csiro.au/research/pulsar/psrcat
}

from the radio observations are the spin-down luminosity of $3.1 \times 10^{36} \mathrm{erg} \mathrm{s}^{-1}$ and the surface magnetic field strength of $7.8 \times 10^{12} \mathrm{G}$, inferred under the assumption of pure magnetic dipole braking. Based on a dispersion measure of $\sim 127 \mathrm{~cm}^{-3} \mathrm{pc}$ (Camilo et al. 2004), a distance of $\sim 2.4 \mathrm{kpc}$ is estimated, according to the Cordes-Lazio NE2001 Galactic Free Electron Density Model $^{2}$.

Here we report the first detection of PSR J1357-6429 in the $\mathrm{X}$-ray range using the XMM-Newton observatory and we present its spectral properties in the $0.5-10 \mathrm{keV}$ energy band. We also made use of two short Chandra observations to confirm the identification and to probe possible spatial extended emissions, taking advantage of the superb angular resolution of the Chandra telescope.

\section{XMM-Newton observation and data analysis}

In this section we present the results obtained with the EPIC instrument on board the XMM-Newton X-ray observatory. EPIC consists of two MOS (Turner et al. 2001) and one pn CCD detectors (Strüder et al. 2001) sensitive to photons with energies between 0.1 and $15 \mathrm{keV}$. All the data reduction was performed using the XMM-Newton Science Analysis Software ${ }^{3}$ (SAS version 7.0). The raw observation data files were processed using standard pipeline tasks (epproc for pn, emproc for MOS data). Response matrices and effective area files were generated with the SAS tasks rmfgen and arfgen.

The observation was carried out on 2005 August 17 and had a duration of $15 \mathrm{ks}$, yielding net exposure times of $11.7 \mathrm{ks}$ in the

\footnotetext{
${ }^{2}$ See http://rsd-www.nrl.navy.mil/7213/lazio/ne_model and references therein.

${ }^{3}$ See http://xmm.vilspa.esa.es/
} 


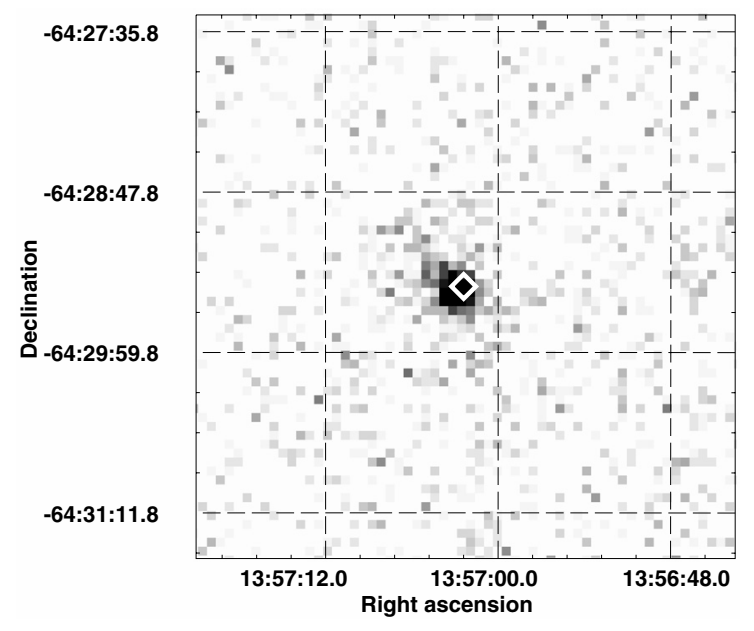

Fig. 1. Field of PSR J1357-6429 as seen by the EPIC cameras in the 0.5-10 keV energy range. The radio pulsar position (Camilo et al. $2004)$ is marked with the white diamond sign. The angular separation of the centroid of the X-ray source (computed using the SAS task emldetect) from the radio pulsar position is $(3.5 \pm 0.6)^{\prime \prime}(1 \sigma$ statistical error). Considering the XMM-Newton absolute astrometric accuracy of $2^{\prime \prime}$ (rms), the X-ray and radio positions are consistent.

pn camera and $14.5 \mathrm{ks}$ in the two MOSs. The pn and the MOSs were operated in Full Frame mode (time resolution: $73.4 \mathrm{~ms}$ and $2.6 \mathrm{~s}$, respectively) and mounted the medium thickness filter. PSR J1357-6429 is clearly detected in the pn and MOS images (see Fig. 1) at the radio pulsar position (Right ascension = $13^{\mathrm{h}} 57^{\mathrm{m}} 02.4^{\mathrm{s}}$, Declination $=-64^{\circ} 29^{\prime} 30.2^{\prime \prime}$ (epoch J2000.0); Camilo et al. 2004). The source spectra were extracted from circular regions centered at the position of PSR J1357-6429. The whole observation was affected by a high particle background that led to the selection of a $20^{\prime \prime}$ radius circle in order to increase the signal-to-noise ratio in the pn detector, particularly sensitive to particle background, and a $40^{\prime \prime}$ radius for both the MOS cameras. The background spectra were extracted from annular regions with radii of $140^{\prime \prime}$ and $220^{\prime \prime}$ for the MOSs, and from two rectangular regions with total area of $\sim 10^{4} \operatorname{arcsec}^{2}$ located on the sides of the source for the pn. We carefully checked that the choice of different background extraction regions does not affect the spectral results. We selected events with pattern 0-4 and pattern 0-12 for the pn and the MOS, respectively. The resulting background subtracted count rates in the $0.5-10 \mathrm{keV}$ energy range were $(4.2 \pm 0.3) \times 10^{-2} \mathrm{cts} \mathrm{s}^{-1}$ in the pn and $(1.9 \pm 0.2) \times 10^{-2} \mathrm{cts} \mathrm{s}^{-1}$ in the two MOS cameras, while the background rate expected in the source extraction regions is about $50 \%$ of these values. The spectra were rebinned to have at least 20 counts in each energy bin. Spectral fits were performed using the XSPEC version 12.3 software ${ }^{4}$.

The spectra from the three cameras were fitted together in the $0.5-10 \mathrm{keV}$ energy range with a power law and with a powerlaw plus blackbody model (see Table 1). The latter model provides a slightly better fit, with less structured residuals (see Fig. 2). Furthermore, considering the distance of $2.5 \mathrm{kpc}$, the interstellar absorption along the line of sight derived with the power-law fit is too low if compared to the typical column density of neutral absorbing gas in that direction of approximately $10^{22} \mathrm{~cm}^{-2}$ (Dickey \& Lockman 1990). The resulting best-fit parameters for the power-law plus blackbody model are photon index $\Gamma=1.4$, blackbody temperature $k_{\mathrm{B}} T=0.16 \mathrm{keV}$, and

\footnotetext{
${ }^{4}$ See http://heasarc.gsfc.nasa.gov/docs/xanadu/xspec/
}

Table 1. Summary of the XMM-Newton spectral results. Errors are at the $90 \%$ confidence level for a single interesting parameter.

\begin{tabular}{ccc}
\hline \hline Parameter & \multicolumn{2}{c}{ Value } \\
& $\mathrm{PL}$ & $\mathrm{PL}+\mathrm{BB}$ \\
\hline$N_{\mathrm{H}}\left(10^{22} \mathrm{~cm}^{-2}\right)$ & $0.14_{-0.06}^{+0.07}$ & $0.4_{-0.2}^{+0.3}$ \\
$\Gamma$ & $1.8_{-0.2}^{+0.3}$ & $1.4 \pm 0.5$ \\
$k_{\mathrm{B}} T(\mathrm{keV})$ & - & $0.16_{-0.04}^{+0.09}$ \\
$R_{B B}{ }^{a}(\mathrm{~km})$ & - & $1.4_{-0.2}^{+2.9}$ \\
Flux $^{b}\left(10^{-13} \mathrm{erg} \mathrm{cm}^{-2} \mathrm{~s}^{-1}\right)$ & 2.3 & 3.6 \\
Blackbody flux $^{b}\left(10^{-13} \mathrm{erg} \mathrm{cm}^{-2} \mathrm{~s}^{-1}\right)$ & - & 1.3 \\
$\chi_{r}^{2} /$ d.o.f. & $1.00 / 72$ & $0.85 / 70$ \\
\hline
\end{tabular}

${ }^{a}$ Radius at infinity assuming a distance of $2.5 \mathrm{kpc}$.

${ }^{b}$ Unabsorbed flux in the $0.5-10 \mathrm{keV}$ energy range.

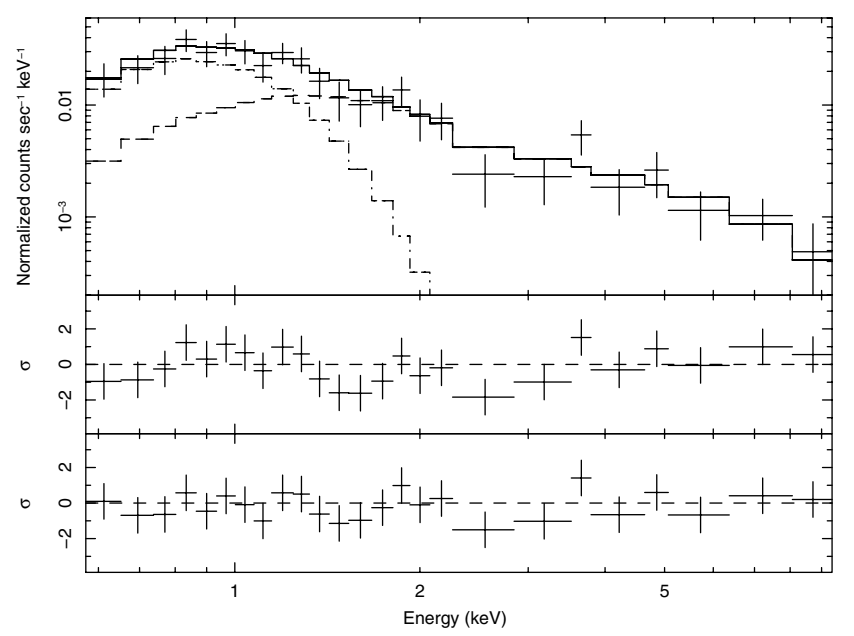

Fig. 2. EPIC pn spectrum of PSR J1357-6429. Top: data and bestfit power-law (dashed line) plus blackbody (dot-dashed line) model. Middle: residuals from the power-law best-fit model in units of standard deviation. Bottom: residuals from the power-law plus blackbody best-fit model in units of standard deviation.

absorption $N_{\mathrm{H}}=4 \times 10^{21} \mathrm{~cm}^{-2}$ with a reduced $\chi^{2}$ of 0.85 for 70 degrees of freedom. The corresponding luminosity in the $0.5-10 \mathrm{keV}$ band is $2.7 \times 10^{32} \mathrm{erg} \mathrm{s}^{-1}$.

Young pulsars are often associated with pulsar wind nebulae: complex structures that arise from the interaction between the particle wind powered by the pulsar and the supernova ejecta or surrounding interstellar medium (see Gaensler \& Slane 2006, for a review). Inspecting the EPIC images in various energy bands, we find only a marginal $(\approx 3 \sigma)$ evidence of diffuse emission, in the $2-4 \mathrm{keV}$ energy band consisting of a faint elongated ( $\sim 20$ arcsec to the north-east, see Fig. 1) structure starting from PSR J1357-6429. We took that excess as an upper limit for a diffuse emission: assuming the same spectrum as the point source, it corresponds to a $2-10 \mathrm{keV}$ luminosity of $\approx 6 \times 10^{31} \mathrm{erg} \mathrm{s}^{-1}$.

For the timing analysis we applied the solar system barycenter correction to the photon arrival times with the SAS task barycen. We searched the data for pulsations around the spin frequency at the epoch of the XMM-Newton observations, predicted assuming the pulse period and the spin-down rate measured with the Parkes radio telescope (Camilo et al. 2004). As glitches and/or deviations from a linear spin-down may alter the period evolution, we searched over a wide period range centered at the value of $\sim 166 \mathrm{~ms}$. We searched for significant periodicities using two methods: a standard folding technique and the Rayleigh statistic. No pulsation were detected near to the 


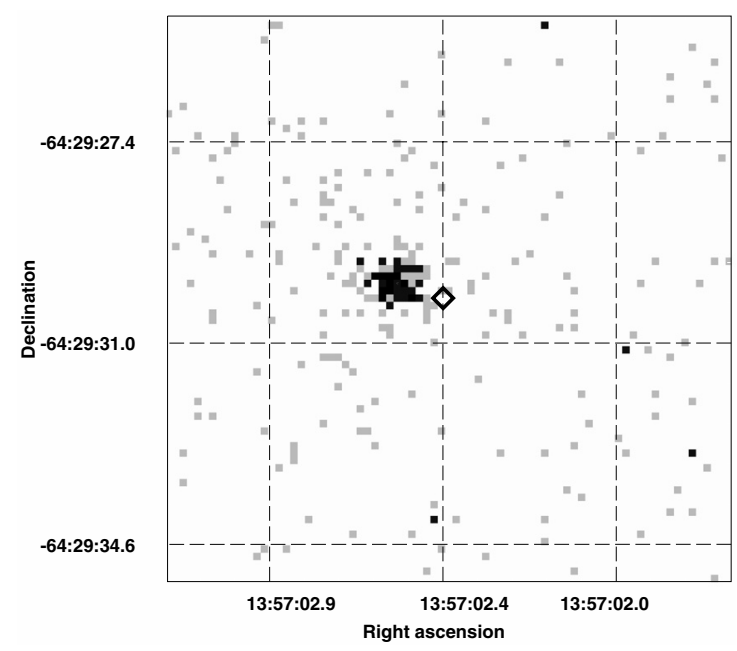

Fig. 3. Chandra $0.08-10 \mathrm{keV}$ HRC-S image centered on the radio pulsar position, marked with a diamond sign (Camilo et al. 2004). The CIAO celldetect routine yields a best-fit position for the X-ray source at an angular distance of $(0.9 \pm 0.2)^{\prime \prime}(1 \sigma$ statistical error $)$ from the radio pulsar. This value consistent with the Chandra pointing accuracy of $0.8^{\prime \prime}$ (99\% confidence level).

predicted frequency with either method but, since the pn timing resolution ( $73 \mathrm{~ms}$ ) allows to only poorly sample the $166 \mathrm{~ms}$ pulsar period, a reliable upper limit on the pulsed fraction cannot be set.

\section{Chandra observations and data analysis}

PSR J1357-6429 was observed by means of the Chandra X-ray Observatory during two exposures of $\sim 17 \mathrm{ks}$ duration each on 2005 November 18 and 19. The observations were carried out with the Spectroscopic array of the High Resolution Camera (HRC-S; Murray et al. 2000) used without transmission gratings. The HRC is a multichannel plate detector sensitive to X-ray over the $0.08-10 \mathrm{keV}$ energy range, although essentially no energy information on the detected photons is available. The HRC-S time resolution is $16 \mu \mathrm{s}$.

We started from "level 1" event data calibrated and made available through the Chandra X-ray Center ${ }^{5}$. The level 1 event files contain all HRC triggers with the position information corrected for instrumental (degap) and aspect (dither) effects. After standard data processing with the Chandra Interactive Analysis of Observations (CIAO ver. 3.3), a point-like source has been clearly detected in both the observations at a position consistent with that of PSR J1357-6429 (see Fig. 3).

For the timing analysis we corrected the data to the solar system barycenter with the CIAO task axbary and then we followed the same procedure described in Sect. 2, but we again did not detect the source pulsation. By folding the light curve of PSR J1357-6429 on the radio frequency and fitting it with a sinusoid, we determine a $90 \%$ confidence level upper limit of $\sim 30 \%$ on the amplitude of a sinusoidal modulation. We stress that this upper limit depends sensitively on data time binning and on the assumed pulse shape.

We used the CIAO task merge_all to generate a combined image of the source. Our main purpose was to search for diffuse structures on scales smaller than the XMM-Newton angular resolution. We compared the radial profile of the

\footnotetext{
5 See http://cxc.harvard.edu/
}

pulsar emission with the Chandra High-Resolution Mirror Assembly point-spread function at $1 \mathrm{keV}$ generated using Chandra Ray Tracer (ChaRT) and Model AXAF Response to $\mathrm{X}$-rays (MARX). We found that the emission we detect from PSR J1357-6429 ( $\sim 100$ counts concentrated within a $\sim 0.5^{\prime \prime}$ radius circle) is consistent with that from a point source.

We used the Chandra data and the PIMMS software ${ }^{6}$ to determine an upper limit on the luminosity of a possible spatial extended emission. The $3 \sigma$ upper limit on a pulsar wind nebula brightness (in counts s ${ }^{-1}$ ) has been estimated as $3(b A)^{1 / 2} \tau^{-1}$, where $b$ is the background surface brightness in counts arcsec ${ }^{-2}$, $A$ is the pulsar wind nebula area, and $\tau$ is the exposure duration. Assuming the interstellar absorption value from the $X M M-N e w t o n$ best-fit model $\left(N_{\mathrm{H}}=0.4 \times 10^{22} \mathrm{~cm}^{-2}\right.$, see Sect. 2$)$ and typical parameters for a pulsar wind nebula (radius of $\sim 2 \times$ $10^{17} \mathrm{~cm}$, that corresponds to $\sim 5^{\prime \prime}$ for a distance of $2.5 \mathrm{kpc}$, and power-law spectrum with photon index $\Gamma=1.6$, see, e.g., Gotthelf 2003), this upper limit corresponds to a $2-10 \mathrm{keV} \mathrm{lu}-$ minosity of $\approx 3 \times 10^{31} \mathrm{erg} \mathrm{s}^{-1}$ for a uniform diffuse nebula. No significant diffuse excess was found even at larger angular scale, but the corresponding upper limit for diffuse emission is less constraining than that derived using the XMM-Newton data.

\section{Discussion}

We have presented the results of the first X-ray observations of PSR J1357-6429 by means of the XMM-Newton and Chandra observatories. The source has been positively detected in all the instruments although, probably due to the low statistics, we could not detect the source pulsation. The high angular resolution Chandra observations favor the picture in which most of the counts belong to a point source. We found that the spectrum is well represented by either a power-law with photon index $\Gamma=1.8_{-0.2}^{+0.3}$ or by a power-law plus blackbody model. In the latter case the best-fit parameters are for the power-law component a photon index $\Gamma=1.4 \pm 0.5$ and, for the blackbody component, radius ${ }^{7}$ of $\sim 1.4_{-0.2}^{+2.9} d_{2.5} \mathrm{~km}$ and temperature corresponding to $k_{\mathrm{B}} T=0.16_{-0.04}^{+0.09} \mathrm{keV}$.

It is generally believed that a combination of emission mechanisms are responsible for the detected X-ray flux from rotationpowered pulsars (see, e.g., Kaspi et al. 2006, for a review). The acceleration of particles in the neutron star magnetosphere generates non thermal radiation by synchrotron and curvature radiation and/or inverse Compton processes, while soft thermal radiation could result by cooling of the surface of the neutron star. A harder thermal component can arise from polar-cap reheating, due to return currents from the outer gap or from close to the polar-cap. The dominant emission mechanism is likely related to the age of the pulsar. In pulsar younger than $\approx 10^{4} \mathrm{yr}$ the strong magnetospheric emission generally prevails over the thermal radiation, making difficult to detect it.

As discussed in Sect. 2, we tend to prefer the power-law plus blackbody spectral model for PSR J1357-6429. The resulting blackbody size of $\sim 1.5 d_{2.5} \mathrm{~km}$ may suggest that the soft emission $(\$ 2 \mathrm{keV})$ is coming from hot spots on the surface due to backflowing particles, rather than from the entire surface. However this hint should be considered with caution, as the surface temperature distribution of a neutron star is most likely non uniform (since the heath conductivity of the crust is higher along the magnetic field lines) and the small and hot blackbody could result from a more complicated distribution of temperature. Moreover,

\footnotetext{
6 See http://heasarc.gsfc.nasa.gov/docs/tools.html

7 We indicate with $d_{\mathrm{N}}$ the distance in units of $\mathrm{N} \mathrm{kpc}$.
} 
currently we lack of reliable models of cooling neutron star thermal emission and thus we cannot exclude that the soft component is emitted from surface layers of the whole neutron star.

To date, thermal emission has been detected in only a few young radio pulsars. Among these, the properties of PSR J1357-6429 are similar to those of the young pulsars Vela (PSR B0833-45; $\tau_{\mathrm{c}}=11 \mathrm{kyr}, P=89 \mathrm{~ms}, E=6.9 \times$ $10^{36} \mathrm{erg} \mathrm{s}^{-1}$, and distance $d \simeq 0.2 \mathrm{kpc}$; Pavlov et al. 2001) and PSR B1706-44 $\left(\tau_{\mathrm{c}}=17.5 \mathrm{kyr}, P=102 \mathrm{~ms}, \dot{E}=3.4 \times\right.$ $10^{36} \mathrm{erg} \mathrm{s}^{-1}$, and $d \simeq 2.5 \mathrm{kpc}$; Gotthelf et al. 2002). Notably, the efficiency in the conversion of the spin-down energy loss into X-ray luminosity for PSR J1357-6429 is $L_{0.5-10 \mathrm{keV}} / \dot{E} \simeq 8 d_{2.5}^{2} \times$ $10^{-5}$, significantly lower than the typical value of $\approx 10^{-3}$ (Becker \& Truemper 1997), and similar to that of PSR B1706-44 $\left(\sim 10^{-4}\right)$ and Vela $\left(\sim 10^{-5}\right)$.

Although a pulsar wind nebula would not came as a surprise for this young and energetic source, we did not find clear evidence of diffuse X-ray emission associated with PSR J1357-6429. However, some known examples of wind nebulae (see Gaensler \& Slane 2006), rescaled to the distance of PSR J1357-6429, would hide below the upper limits derived from the XMM-Newton and Chandra data.

New deeper exposures using XMM-Newton or Chandra would help determine if a thermal component is present in the emission of PSR J1357-6429 as our spectral analysis suggests, and possibly detect a pulsed emission. High sensitivity observations would also serve to address the issue of the presence of a pulsar wind nebula. Although there is not any EGRET $\gamma$-ray source coincident with PSR J1357-6429 (Hartman et al. 1999), young neutron stars and their nebulae are often bright $\gamma$-ray sources and PSR J1357-6429 in particular, given its high "spindown flux" $\dot{E} / d^{2}$ and similarity with Vela and PSR B1706-44, is likely to be a good target for the upcoming AGILE and GLAST satellites and the ground based Cherenkov air showers telescopes.

Acknowledgements. This work is based on data from observations with XMM-Newton, an ESA science mission with instruments and contributions directly funded by ESA member states and NASA. We also used data from the Chandra X-ray Observatory Center, which is operated by the Smithsonian Astrophysical Observatory Center on behalf of NASA. The authors thank the anonymous referee for helpful comments and acknowledge the support of the Italian Space Agency and the Italian Ministry for University and Research.

\section{References}

Becker, W., \& Truemper, J. 1997, A\&A, 326, 682

Camilo, F., Manchester, R. N., Lyne, A. G., et al. 2004, ApJ, 611, L25

Dickey, J. M., \& Lockman, F. J. 1990, ARA\&A, 28, 215

Duncan, A. R., Stewart, R. T., Haynes, R. F., \& Jones, K. L. 1997, MNRAS, 287, 722

Gaensler, B. M., \& Slane, P. O. 2006, ARA\&A, 44, 17

Gotthelf, E. V. 2003, ApJ, 591, 361

Gotthelf, E. V., Halpern, J. P., \& Dodson, R. 2002, ApJ, 567, L125

Hartman, R. C., Bertsch, D. L., Bloom, S. D., et al. 1999, ApJS, 123, 79

Kaspi, V. M., Roberts, M. S. E., \& Harding, A. K. 2006, in Compact stellar X-ray sources, ed. W. H. G. Levin, \& M. van der Klis (Cambridge: Cambridge University Press), 279

Lorimer, D. R., Faulkner, A. J., Lyne, A. G., et al. 2006, MNRAS, 372, 777

Manchester, R. N., Hobbs, G. B., Teoh, A., \& Hobbs, M. 2005, AJ, 129, 1993

Murray, S. S., Austin, G. K., Chappell, J. H., et al. 2000, in X-Ray Optics, Instruments, and Missions III, ed. J. E. Truemper, \& B. Aschenbach, Proc. SPIE, 4012, 68

Pavlov, G. G., Zavlin, V. E., Sanwal, D., Burwitz, V., \& Garmire, G. P. 2001, ApJ, 552, L129

Strüder, L., Briel, U., Dennerl, K., et al. 2001, A\&A, 365, L18

Taylor, J. H., Manchester, R. N., \& Lyne, A. G. 1993, ApJS, 88, 529

Turner, M. J. L., Abbey, A., Arnaud, M., et al. 2001, A\&A, 365, L27 